\title{
2581. Simultaneous excitation and parameter identification for non-linear structural systems
}

\author{
Zhimin Wan', Ting Wang ${ }^{2}$, Qibai Huang ${ }^{3}$, Weiguang Zheng ${ }^{4}$, Feng Gu ${ }^{5}$ \\ ${ }^{1,3,}{ }^{4}$ School of Mechanical Science and Engineering, Huazhong University of Science and Technology, \\ Wuhan 430074, China \\ $1,3,4$ Hubei Institute of Specialty Vehicle, Suizhou 441300, China \\ ${ }^{1,5}$ School of Vehicle and Transportation Engineering, Nantong Vocational University, \\ Nantong 226000, China \\ ${ }^{2}$ School of Mechanical Engineering, Nantong Vocational University, Nantong 226000, China \\ ${ }^{3}$ Corresponding author \\ E-mail: 1'zhiminwan@hust.edu.cn, ${ }^{2}$ lwxting@hust.edu.cn, ${ }^{3}$ qibaihuang@hust.edu.cn, \\ 4weiguang.zheng@foxmail.com,5feng.gu@foxmail.com
}

Received 18 August 2016; received in revised form 6 February 2017; accepted 29 March 2017 DOI https://doi.org/10.21595/jve.2017.17576

Check for updates

Abstract. In this paper, an algorithm is proposed for simultaneous excitation and parameter identification for non-linear system in state space. The algorithm is based on the sequential application of extended Kalman estimator for non-linear structural parameters and the weighted least squares estimation for unknown excitations. The state and parameter are reformed into the augmented state, and the state space equations are non-linear associated with the augmented state. With the first-order Taylor expansion for nonlinear system and approximately linear minimum-variance unbiased estimation, a recursive algorithm is derived where the identification of the augmented state and the excitation are interconnected. Two numerical examples which identify uncertain parameters of a 3-DOF Duffing-type system and a four-story hysteretic shear-beam building subject to unknown random excitation respectively, are conducted to demonstrate the effectiveness of the proposed approach.

Keywords: parameter identification, excitation identification, extended Kalman filter, non-linear system.

\section{Introduction}

Parameter identification is an important research topic in structural dynamics, which can be easily applied for structural damage identification, model updating and health monitoring. Various analysis approaches for parameter identification have been presented in the literatures [1, 2]. The popularity of the currently available parameter identification approaches is suitable for linear structural systems. However, structural non-linearity widely exists in engineering structures. For example, in civil engineering filed, when damage occurs in concrete structures, the open and close of cracks under dynamic excitation are typical non-linear process which leads to the hysteretic performance of the structures. Therefore, it is necessary to develop the novel approaches of parameter identification for non-linear structural systems. Masri [3-5] is among the pioneers for the developments of various methods for the identification of non-linear structural systems from measured vibration data. Additionally, various filter algorithms, such as extended Kalman filter (EKF) [6-11], $H_{\infty}$ filter [12], unscented Kalman filter (UKF) [13], Monte Carlo filter [14] and particle filter $(\mathrm{PF})[15,16]$, have been also developed for parameter identification for non-linear structural systems.

In the filter algorithms above, all the external excitations should be available from sensor measurements. Unfortunately, there is no straightforward way to measure the excitations on a structure because the introduction of dedicated force cells requires alteration to the structure to locate the sensor in the force path, which is unwanted and unpractical. And also, sensors may not be installed in the health monitoring system to measure all the excitations, such as earthquake. Therefore, it is essential to develop the algorithms for simultaneous excitation and parameter identification. Yang et al. [17] proposed a modified EKF under unknown excitation inputs, 
referred to as EKF-UI, for the identification of structural parameters as well as the unknown excitations. Lei et al. [18] deduced the EKF-UI algorithm again by the least-squares estimation, and pointed that the analytical recursive solutions by the original EKF-UI were obtained by relatively complex mathematical derivations. Lei et al. [19] also adopted the simplified EKF-UI algorithm for the identification of non-linear structural parameter. But in the simplified EKF-UI algorithm, the prior probability density functions (PDFs) of states at $t+1$ time are taken as the posterior PDFs of states at $t+1$ time, which may cause the confusion of concepts in Bayesian framework.

In 2007, Gillijns and De Moor [20] developed a recursive optimal filter of joint state/input estimation for linear systems with direct transmission, which was originally proposed for optimal control applications. The identified input and state by this filter are optimal in a minimum-variance unbiased sense. In this paper, a novel method of simultaneous excitation and parameter identification based on the filter proposed by Gillijn and De Moor (GDF) for non-linear structural systems is developed. The standard GDF algorithm is only suitable for joint state/input identification for linear systems. In this method, it is extended for non-linear systems by the linearization idea of EKF. The uncertain parameters and the states are considered as the augmented states to be identified. Thus, the novel state transmission and measurement equations are non-linear, which are linearized by the first-order Taylor expansions. The proposed extended GDF (EGDF) has the same structure of the standard GDF algorithm, including three steps: input identification, measurement update and time update. The main difference between them is the sensitivity matrices of the non-linear state-space equations.

This paper proceeds as follows. Section 2 presents the standard GDF algorithm for joint state/input identification. In Section 3, the non-linear identification model of the coupled state/input/parameter is firstly built, and then the proposed EGDF method is derived in detail. In Section 4, two numerical examples are conducted to demonstrate the effectiveness of the EGDF method. Finally, the conclusion is drawn based on the current study.

\section{Joint state/input identification}

\subsection{Discrete-time state-space model of structural dynamics}

The general equation of motion of a damped structure with $n$ DOFs can be written as:

$$
\mathbf{M} \ddot{\mathbf{p}}(t)+\mathbf{C} \dot{\mathbf{p}}(t)+\mathbf{K} \mathbf{p}(t)=\mathbf{B}_{u} \mathbf{u}(t),
$$

where $\mathbf{M}, \mathbf{C}$ and $\mathbf{K} \in \mathbb{R}^{n \times n}$ are the mass, damping and stiffness matrices of the structure, respectively; $\ddot{\mathbf{p}}(t), \dot{\mathbf{p}}(t), \mathbf{p}(t) \in \mathbb{R}^{n}$ are, respectively, the nodal acceleration, velocity and displacement vectors of the structure; $\mathbf{u}(t) \in \mathbb{R}^{m}$ is the force vector and $\mathbf{B}_{u} \in \mathbb{R}^{n \times m}$ is the influence matrix associated with $\mathbf{u}(t)$.

The second-order equation of motion Eq. (1) can be transformed into a first-order continuous-time state equation as:

$\dot{\mathbf{x}}(t)=\mathbf{A}_{c} \mathbf{x}(t)+\mathbf{B}_{c} \mathbf{u}(t)$

where $\mathbf{x}(t) \in \mathbb{R}^{2 n \times 2 n}$ is the state vector, and the system matrices together with $x(t)$ are defined as:

$$
\mathbf{A}_{c}=\left[\begin{array}{cc}
{[\mathbf{0}]} & \mathbf{I} \\
-\mathbf{M}^{-1} \mathbf{K} & -\mathbf{M}^{-1} \mathbf{C}
\end{array}\right], \quad \mathbf{B}_{c}=\left[\begin{array}{c}
{[\mathbf{0}]} \\
\mathbf{M}^{-1} \mathbf{B}_{u}
\end{array}\right], \quad \mathbf{x}(t)=\left[\begin{array}{c}
\mathbf{p}(t) \\
\dot{\mathbf{p}}(t)
\end{array}\right] .
$$

Assuming that only $s$ acceleration signals are measured (i.e. $y(t) \in \mathbb{R}^{s}$ ), the measurement equation can be written as the following state-space form: 
$\mathbf{y}(t)=\mathbf{H x}(t)+\mathbf{D u}(t)$

With the output influence matrix $\mathbf{H} \in \mathbb{R}^{s \times n}$ and direct transmission matrix $\mathbf{D} \in \mathbb{R}^{s \times m}$ defined as:

$$
\mathbf{H}=\left[\begin{array}{ll}
-\mathbf{H}_{0} \mathbf{M}^{-1} \mathbf{K} & -\mathbf{H}_{0} \mathbf{M}^{-1} C
\end{array}\right], \quad \mathbf{D}=\left[\mathbf{H}_{0} \mathbf{M}^{-1} \mathbf{B}_{u}\right],
$$

where the $\mathbf{H}_{0}$ is the selection matrix of appropriate dimension for accelerations and composed by zeros and ones.

Let the symbol $\Delta t$ denote the time step size, thus the continuous-time state-space model of Eqs. (2) and (4) can be transformed into the following discrete-time form using a sampling rate of $1 / \Delta t$ :

$\mathbf{x}_{k+1}=\mathbf{A} \mathbf{x}_{k}+\mathbf{B} \mathbf{u}_{k}$,

$\mathbf{y}_{k}=\mathbf{H} \mathbf{x}_{k}+\mathbf{D u}_{k}$,

where:

$\mathbf{x}_{k}=\mathbf{x}(k \Delta \mathrm{t}), \quad \mathbf{y}_{k}=\mathbf{y}(k \Delta \mathrm{t}), \quad k=1,2,3$,

$\mathbf{A}=e^{\mathbf{A}_{c} \Delta t}, \quad \mathbf{B}=\left(e^{\mathbf{A}_{c} \Delta t}-\mathbf{I}\right) \mathbf{A}_{c}^{-1} \mathbf{B}_{c}$,

\subsection{The GDF algorithm}

Considering the system and measurement noise, the linear structural system can be rewritten as:

$\mathbf{x}_{k+1}=\mathbf{A} \mathbf{x}_{k}+\mathbf{B} \mathbf{u}_{k}+\mathbf{w}_{k}=f_{k}\left(\mathbf{x}_{k}, u_{k}\right)+\mathbf{w}_{k}$,

$\mathbf{y}_{k}=\mathbf{H} \mathbf{x}_{k}+\mathbf{D} \mathbf{u}_{k}+\mathbf{v}_{k}=h_{k}\left(\mathbf{x}_{k}, u_{k}\right)+\mathbf{v}_{k}$.

The system noise vector $\mathbf{w}_{\mathbf{k}} \in \mathbb{R}^{n}$ and measurement noise vector $\mathbf{v}_{k} \in \mathbb{R}^{m}$ are assumed to be mutually uncorrelated, zero-mean, white random signal with known covariance matrices, $\mathbf{G}_{k}=E\left\{\mathbf{w}_{k} \mathbf{w}_{l}^{T}\right\}$ and $\mathbf{R}_{k}=E\left\{\mathbf{v}_{k} \mathbf{v}_{l}^{T}\right\}$ respectively ( $E$ represents the expectation value). Results are easily generalized to the case where $\mathbf{w}_{\mathbf{k}}$ and $\mathbf{v}_{k}$ are correlated [21]. If the inputs $\mathbf{u}_{k}$ in Eqs. (10) and (11) are unknown, then the two equations represent the linear dynamic system with direct feedthrough. In the field of system control, Gillijns and De Moor proposed an optimal recursive filter of the direct feedthrough case for joint input/state identification [20]. In this filter, a state estimate $\mathbf{x}_{k \mid l}$ is defined as an estimate of $\mathbf{x}_{k}$ given $\left\{\mathbf{y}_{0}, \mathbf{y}_{1}, \ldots, \mathbf{y}_{l}\right\}$ and its error covariance matrix $\mathbf{P}_{k \mid l}^{x}$ as $E\left[\left(\mathbf{x}_{k}-\mathbf{x}_{k \mid l}\right)\left(\mathbf{x}_{k}-\mathbf{x}_{k \mid l}\right)^{T}\right]$. An initial unbiased state estimate $\mathbf{x}_{0 \mid-1}$ and its covariance matrix $\mathbf{P}_{0 \mid-1}^{x}$ are assumed known.

The GDF algorithm computes the unknown input and state in a recursive procedure which includes three steps: the input identification, the measurement update and the time update. The GDF algorithm is listed in Table 1.

In Ref. [22], the GDF is applied in linear structural dynamics for joint input/response identification successfully. However, it is not suitable for non-linear dynamic systems. In Section 3, the GDF method is extended for non-linear dynamic system to solve the problem of coupled state/input/parameter identification.

\section{Coupled state/input/parameter identification}

In this section, uncertain structure with unknown structural parameters is considered to identify both state and input. Therefore, a novel difficult problem of coupled state/input/parameter identification is presented for more common engineering applications. 
Table 1. The algorithm of GDF

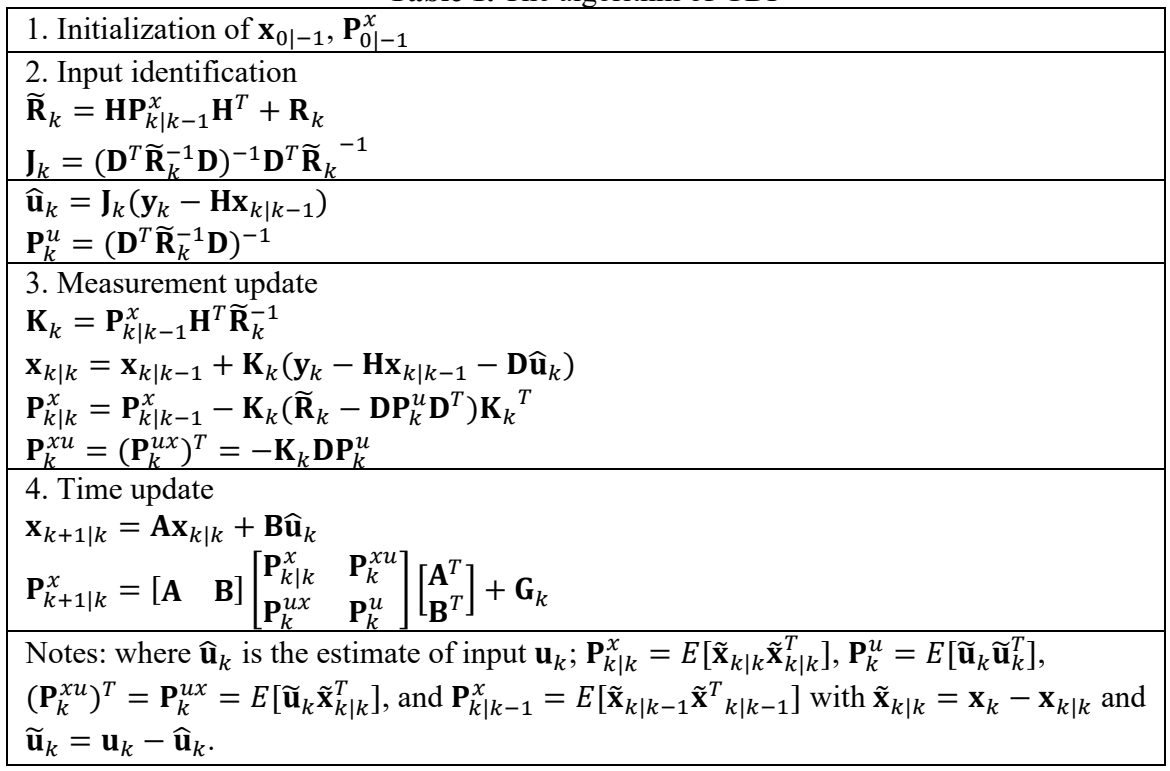

\subsection{Non-linear identification model of the coupled state/input/parameter identification}

Assuming in dynamics Eq. (1) that only $\mathbf{K}$ and $\mathbf{C}$ contain the uncertain parameters $\alpha$ to be identified, the original state vector $\mathbf{x}$ can be extended to be the augmented state vector as:

$z(t)=\left[\begin{array}{c}\mathbf{x}(t) \\ \alpha\end{array}\right]$

where $\alpha=\left[\begin{array}{llll}\alpha_{1} & \alpha_{2} & \cdots & \alpha_{\alpha}\end{array}\right]^{T} \in \mathbb{R}^{\alpha}$ is the parameter vector. Therefore, the augmented state transmission and measurement equations can be obtained as:

$\dot{z}(t)=\left[\begin{array}{l}\dot{\mathbf{x}}(t) \\ \dot{\mathbf{a}}\end{array}\right]=\left[\begin{array}{l}\dot{\mathbf{p}}(t) \\ \ddot{\mathbf{p}}(t) \\ {[\mathbf{0}]}\end{array}\right]=\left[\begin{array}{c}\dot{\mathbf{p}}(t) \\ -\mathbf{M}^{-1} \mathbf{K} \mathbf{p}(t)-\mathbf{M}^{-1} \mathbf{C} \dot{\mathbf{p}}(t)+\mathbf{M}^{-1} \mathbf{B}_{u} \mathbf{u}(t) \\ {[0]}\end{array}\right] \equiv f^{c}(\mathbf{z}(t), \mathbf{u}(t))$,

$\mathbf{y}(t)=\mathbf{H x}(t)+\mathbf{D u}(t) \equiv h(\mathbf{z}(t))+\mathbf{D u}(t)$

The parameters are assumed to be constant, i.e. $\dot{\alpha}=[\mathbf{0}]$.

The above continuous-time state-space equations can be transformed into the following form as:

$\mathbf{z}_{k+1}=f_{k}\left(\mathbf{z}_{k}, \mathbf{u}_{k}\right)+\mathbf{w}_{k}, \quad k=1,2 \ldots, T$,

$\mathbf{y}_{k}=h_{k}\left(\mathbf{z}_{k}\right)+\mathbf{D}_{k} \mathbf{u}_{k}+\mathbf{v}_{k}, \quad k=1,2, \ldots, T$.

Unlike Eqs. (10) and (11), the functions $f$ and $h$ are both non-linear. The standard GDF method cannot be adopted to handle this case of non-linear identification. In the research field of system control, the standard KF was presented for identifying the state of linear dynamic systems. The EKF algorithm was then developed for identifying the state of non-linear dynamic systems by continually updating a linearization around the previous state estimate, starting with an initial guess, which is a non-linear variation on the standard KF based on the first-order Taylor approximation. In the following section, the linearization idea of the EKF method is adopted to extend the standard GDF method for non-linear dynamic systems. 


\subsection{The extended GDF algorithm}

In this coupled state/input/parameter identification problem, the augmented state $\mathbf{z}_{k}$ (including state and uncertain parameters) and identified forces $\mathbf{u}_{k}$ are both unknown. Therefore, we have the first-order Taylor series expansions for the two non-linear system matrices:

$$
\begin{aligned}
& f_{k}\left(\mathbf{z}_{k}, \mathbf{u}_{k}\right)=f_{k}\left(E\left(\mathbf{z}_{k}\right), E\left(\mathbf{u}_{k}\right)\right)+\nabla_{z} f_{k} \cdot\left(\mathbf{z}_{k}-E\left(\mathbf{z}_{k}\right)\right)+\nabla_{\mathbf{u}} f_{k} \cdot\left(\mathbf{u}_{k}-E\left(\mathbf{u}_{k}\right)\right)+\text { HOT, } \\
& h_{k}\left(\mathbf{z}_{k}\right)=h_{k}\left(E\left(\mathbf{z}_{k}\right)\right)+\nabla_{z} h_{k} \cdot\left(\mathbf{z}_{k}-E\left(\mathbf{z}_{k}\right)\right)+H O T,
\end{aligned}
$$

where $\nabla_{z} f_{k}, \nabla_{u} f_{k}$ and $\nabla_{z} h_{k}$ are the sensitivity matrices, and HOT stands for the high-order terms.

Using the Taylor expansions, the Eqs. (17) and (18) can be approximated in the following linear form:

$$
\begin{aligned}
\mathbf{z}_{k} & =f_{k-1}\left(\mathbf{z}_{k-1}, \mathbf{u}_{k-1}\right)+\mathbf{w}_{k-1}=f_{k-1}\left(E\left(\mathbf{z}_{k-1}\right), E\left(\mathbf{u}_{k-1}\right)\right) \\
& +\nabla_{\mathbf{z}} f_{k-1} \cdot\left(\mathbf{z}_{k-1}-E\left(z_{k-1}\right)\right)+\nabla_{\mathbf{u}} f_{k-1} \cdot\left(\mathbf{u}_{k-1}-E\left(\mathbf{u}_{k-1}\right)\right)+\mathbf{w}_{k-1}+H O T \\
& \approx \nabla_{\mathbf{z}} f_{k-1} \cdot \mathbf{z}_{k-1}+\nabla_{\mathbf{u}} f_{k-1} \cdot \mathbf{u}_{k-1}+f_{k-1}\left(E\left(\mathbf{z}_{k-1}\right), E\left(\mathbf{u}_{k-1}\right)\right)-\nabla_{\mathbf{z}} f_{k-1} \cdot E\left(\mathbf{z}_{k-1}\right) \\
& -\nabla_{u} f_{k-1} \cdot E\left(u_{k-1}\right)+w_{k-1}=z_{k}^{l i n} \\
\mathbf{y}_{k} & =h_{k}\left(E\left(\mathbf{z}_{k}\right)\right)+\nabla_{\mathbf{z}} h_{k} \cdot\left(\mathbf{z}_{k}-E\left(\mathbf{z}_{k}\right)\right)+H O T+\mathbf{D}_{k} \mathbf{u}_{k}+\mathbf{v}_{k} \\
& \left.\approx \nabla_{\mathbf{z}} h_{k} \cdot \mathbf{z}_{k}+\mathbf{D}_{k} \mathbf{u}_{k}+h_{k}\left(E\left(\mathbf{z}_{k}\right)\right)-\nabla_{\mathbf{z}} h_{k} \cdot E\left(\mathbf{z}_{k}\right)\right)+\mathbf{v}_{k}=\mathbf{y}_{k}^{l i n} .
\end{aligned}
$$

Then, the prior expectation of the augmented state vector $\mathbf{z}_{k}$ has the approximately linear form as:

$$
\begin{aligned}
& \mathbf{z}_{k \mid k-1} \equiv E\left(\mathbf{z}_{k} \mid \mathbf{Y}_{k-1}\right) \approx E\left(f_{k-1}\left(E\left(\mathbf{z}_{k-1}\right), E\left(\mathbf{u}_{k-1}\right)\right)+\nabla_{\mathbf{z}} f_{k-1} \cdot\left(\mathbf{z}_{k-1}-E\left(\mathbf{z}_{k-1}\right)\right)\right. \\
& \left.+\nabla_{\mathbf{u}} f_{k-1} \cdot\left(\mathbf{u}_{k-1}-E\left(\mathbf{u}_{k-1}\right)\right)+\mathbf{w}_{k-1} \mid \mathbf{Y}_{k-1}\right)=f_{k-1}\left(\mathbf{z}_{k-1 \mid k-1}, \widehat{\mathbf{u}}_{k-1}\right)=\mathbf{z}_{k \mid k-1}^{l i n}
\end{aligned}
$$

Using the three approximately linear Eqs. (19)-(21), the proposed extended GDF (EGDF) algorithm can be derived referring to Ref. [20]. The EGDF algorithm is shown in Table 2. With the algorithm, the augmented state $\mathbf{z}$ and the excitation $\mathbf{u}$ can be simultaneously identified.

Table 2. The algorithm of EGDF

\begin{tabular}{|l|}
\hline 1. Initialization of $\mathbf{z}_{0 \mid-1}, \mathbf{P}_{0 \mid-1}^{Z}$ \\
\hline 2. Input identification \\
$\widetilde{\mathbf{R}}_{k}=\nabla_{z} h_{k} \cdot \mathbf{P}_{k \mid k-1}^{Z} \cdot\left(\nabla_{z} h_{k}\right)^{T}+\mathbf{R}_{k}$ \\
$\mathbf{J}_{k}=\left(\mathbf{D}_{k}^{T} \widetilde{\mathbf{R}}_{k}^{-1} \mathbf{D}_{k}\right)^{-1} \mathbf{D}_{k}^{T} \widetilde{\mathbf{R}}_{k}^{-1}$ \\
$\widehat{\mathbf{u}}_{k}=\mathbf{J}_{k}\left(\mathbf{y}_{k}-h_{k}\left(\mathbf{z}_{k \mid k-1}\right)\right)$ \\
$\mathbf{P}_{k}^{u}=\left(\mathbf{D}_{k}^{T} \widetilde{\mathbf{R}}_{k}^{-1} \mathbf{D}_{k}\right)^{-1}$ \\
\hline 3. Measurement update \\
$\mathbf{K}_{k}=\mathbf{P}_{k \mid k-1}^{z}\left(\nabla_{z} h_{k}\right)^{T} \widetilde{\mathbf{R}}_{k}^{-1}$ \\
$\mathbf{z}_{k \mid k}=\mathbf{z}_{k \mid k-1}+\mathbf{K}_{k}\left(\mathbf{y}_{k}-h_{k}\left(z_{k \mid k-1}\right)-\mathbf{D}_{k} \widehat{\mathbf{u}}_{k}\right)$ \\
$\mathbf{P}_{k \mid k}^{Z}=\mathbf{P}_{k \mid k-1}^{Z}-\mathbf{K}_{k}\left(\widetilde{\mathbf{R}}_{k}-\mathbf{D}_{k} \mathbf{P}_{k}^{u} \mathbf{D}_{k}{ }^{T}\right) \mathbf{K}_{k}^{T}$ \\
$\mathbf{P}_{k}^{Z u}=\left(\mathbf{P}_{k}^{u z}\right)^{T}=-\mathbf{K}_{k} \mathbf{D}_{k} \mathbf{P}_{k}^{u}$ \\
\hline 4. Time update \\
$\mathbf{z}_{k+1 \mid k}=f_{k}\left(\mathbf{z}_{k \mid k}, \widehat{\mathbf{u}}_{k}\right)$ \\
$\mathbf{P}_{k+1 \mid k}^{Z} \equiv E\left(\tilde{z}_{k+1 \mid k} \tilde{z}^{T}{ }_{k+1 \mid k}\right)=\left[\begin{array}{ll}\nabla_{z} f_{k} & \nabla_{u} f_{k}\end{array}\right]\left[\begin{array}{ll}\mathbf{P}_{k \mid k}^{Z} & \mathbf{P}_{k}^{z u} \\
\mathbf{P}_{k}^{u z} & \mathbf{P}_{k}^{u}\end{array}\right]\left[\begin{array}{l}\left(\nabla_{z} f_{k}\right)^{T} \\
\left(\nabla_{u} f_{k}\right)^{T}\end{array}\right]+\mathbf{G}_{k}$ \\
\hline
\end{tabular}

Comparing the EGDF algorithm with the standard GDF algorithm, it is found that the two algorithms are very similar, and the only differences are the three sensitivity matrices of $\nabla_{\mathbf{z}} f_{k}$, $\nabla_{\mathbf{u}} f_{k}$ and $\nabla_{\mathbf{z}} h_{k}$. The three matrices $\nabla_{\mathbf{z}} f_{k}, \nabla_{\mathbf{u}} f_{k}$ and $\nabla_{\mathbf{z}} h_{k}$ in EGDF have the same functions as the matrices $\mathbf{A}_{k}, \mathbf{B}_{k}$ and $\mathbf{H}_{k}$ in standard GDF respectively. 
The three important sensitivity matrices $\nabla_{\mathbf{z}} f_{k}, \nabla_{\mathbf{u}} f_{k}$ and $\nabla_{\mathbf{z}} h_{k}$ are derived one by one in the following description. Using the first-order Taylor series expansion, Eq. (13) can be rewritten as:

$\dot{\mathbf{z}}(t)=\mathbf{A}_{f} \mathbf{z}(t)+f^{c}(\mathbf{z}(t), \mathbf{u}(t))-\mathbf{A}_{f} \mathbf{z}(t)$

where $A_{f}$ is a gradient matrix with the value as:

$$
\begin{aligned}
\mathbf{A}_{f} & =\left.\frac{\partial f^{c}}{\partial \mathbf{z}}\right|_{\mathbf{z = \mathbf { z } _ { k | k }}} \mathbf{I} \\
& =\left[\begin{array}{cccccc}
{[\mathbf{0}]} & \mathbf{I} & {[\mathbf{0}]} & \cdots & {[\mathbf{0}]} \\
-\mathbf{M}^{-1} \mathbf{K} & -\mathbf{M}^{-1} \mathbf{C} & -\mathbf{M}^{-1} \frac{\partial \mathbf{K}}{\partial \alpha_{1}} \mathbf{p}_{k}-\mathbf{M}^{-1} \frac{\partial C}{\partial \alpha_{1}} \dot{\mathbf{p}}_{k} & \cdots & -\mathbf{M}^{-1} \frac{\partial \mathbf{K}}{\partial \alpha_{\alpha}} \mathbf{p}_{k}-\mathbf{M}^{-1} \frac{\partial \mathbf{C}}{\partial \alpha_{\alpha}} \dot{\mathbf{p}}_{k} \\
{[\mathbf{0}]} & {[\mathbf{0}]} & {[\mathbf{0}]} & \cdots & {[\mathbf{0}]}
\end{array}\right] .
\end{aligned}
$$

The quantity $e^{-\mathbf{A}_{f} t}$ is multiplied on both sides of Eq. (22) and integrating the new equation in time interval $\left[\begin{array}{ll}t_{0} & t\end{array}\right]$ :

$$
\begin{aligned}
& e^{-\boldsymbol{A}_{f} t} \dot{\mathbf{z}}(t)=e^{-\mathbf{A}_{f} t} \mathbf{A}_{f} \mathbf{z}(t)+e^{-\boldsymbol{A}_{f} t}\left(f^{c}(\mathbf{z}(t), \boldsymbol{u}(t))-\mathbf{A}_{f} \mathbf{z}(t)\right), \\
& \int_{t_{0}}^{t} e^{-\mathbf{A}_{f} \tau}\left(\dot{\mathbf{z}}(\tau)-\mathbf{A}_{f} \mathbf{z}(\tau)\right) d \tau=\int_{t_{0}}^{t} e^{-\mathbf{A}_{f} t}\left(f^{c}(\mathbf{z}(t), \mathbf{u}(t))-\mathbf{A}_{f} \mathbf{z}(t)\right) d \tau, \\
& \mathbf{z}(t)=\mathbf{z}\left(t_{0}\right) e^{\mathbf{A}_{f}\left(t-t_{0}\right)}+\int_{t_{0}}^{t} e^{\mathbf{A}_{f}(t-\tau)}\left(f^{c}(\mathbf{z}(\tau), \mathbf{u}(\tau))-\mathbf{A}_{f} \mathbf{z}(\tau)\right) d \tau .
\end{aligned}
$$

According to Eq. (26), the discrete-time state transmission equation is obtained:

$\mathbf{z}_{k+1}=\nabla_{\mathbf{z}} f_{k} \cdot \mathbf{z}_{k}+\overline{\mathbf{U}}$

where:

$$
\begin{aligned}
& \nabla_{\mathbf{z}} f_{k}=\left.\nabla_{\mathbf{z}} f_{k}\right|_{\mathbf{z}=\mathbf{z}_{k \mid k}}=e^{\mathbf{A}_{f} \Delta t}=\mathbf{I}+\mathbf{A}_{f} \Delta t+\left(\mathbf{A}_{f} \Delta t\right)^{2} / 2 !+\cdots, \quad\left(\Delta t=t_{k+1}-t_{k}\right), \\
& \overline{\mathbf{U}}=\int_{t_{k}}^{t_{k+1}} e^{\mathbf{A}_{f}\left(t_{k+1}-\tau\right)}\left(f^{c}\left(\mathbf{z}_{k}, \mathbf{u}_{k}\right)-\mathbf{A}_{f} \mathbf{z}_{k}\right) d \tau .
\end{aligned}
$$

For the derivation of the matrix $\nabla_{\mathbf{u}} f_{k}$, Eq. (13) can be rewritten in another form as:

$\dot{\mathbf{z}}(t)=\left[\begin{array}{c}\dot{\mathbf{p}}(t) \\ -\mathbf{M}^{-1} \mathbf{K p}(t)-\mathbf{M}^{-1} \mathbf{C} \dot{\mathbf{p}}(t) \\ {[\mathbf{0}]}\end{array}\right]+\left[\begin{array}{c}{[\mathbf{0}]} \\ \mathbf{M}^{-1} \mathbf{B}_{u} \mathbf{u}(t) \\ {[\mathbf{0}]}\end{array}\right]=f^{A}(\mathbf{z}(t))+\mathbf{B}^{c} \mathbf{u}(t)$,

where $f^{\mathbf{A}}(\mathbf{z}(t))=\left[\begin{array}{c}\dot{\mathbf{p}}(t) \\ -\mathbf{M}^{-1} \mathbf{K} \mathbf{p}(t)-\mathbf{M}^{-1} \mathbf{C} \dot{\mathbf{p}}(t) \\ {[\mathbf{0}]}\end{array}\right]$ and $\mathbf{B}^{c}=\left[\begin{array}{c}{[\mathbf{0}]} \\ \mathbf{M}^{-1} \mathbf{B}_{u} \mathbf{u}(t) \\ {[\mathbf{0}]}\end{array}\right]$. Eq. (30) can be rearranged as:

$\dot{\mathbf{z}}(t)=\mathbf{A}_{f} \mathbf{z}(t)+\left(f^{\mathbf{A}}(\mathbf{z}(t))-\mathbf{A}_{f} \mathbf{z}(t)\right)+\mathbf{B}^{c} \mathbf{u}(t)$.

The following derivation procedure is the same as the procedure of the matrix $\nabla_{\mathbf{z}} f_{k}$. The quantity $e^{-\mathbf{A}_{f} t}$ is multiplied on both sides of Eq. (31) and integrating the new equation in time interval $\left[\begin{array}{ll}t_{0} & t\end{array}\right]$ : 
$\mathbf{z}(t)=\mathbf{z}\left(t_{0}\right) e^{\mathbf{A}_{f}\left(t-t_{0}\right)}+\int_{t_{0}}^{t} e^{\mathbf{A}_{f}\left(t-t_{0}\right)}\left(f^{\mathbf{A}}(\mathbf{z}(t))-\mathbf{A}_{f} \mathbf{z}(t)+\mathbf{B}^{c} \mathbf{u}(t)\right) d \tau$

Supposing that the external force $\mathbf{u}(t)$ in time step of integration is constant, and discretizing the Eq. (32), we can obtain:

$\nabla_{\mathbf{u}} f_{k}=\left.\nabla_{\mathbf{u}} f\right|_{\mathbf{z}=\mathbf{z}_{k \mid k}}=\left(e^{\mathbf{A}_{f} \Delta t}-I\right)\left(\mathbf{A}_{f}\right)^{-1} \mathbf{B}^{c}$.

Comparing with the derivation of matrices $\nabla_{\mathbf{z}} f_{k}$ and $\nabla_{\mathbf{u}} f_{k}$, the sensitivity matrix of measurement $\nabla_{\mathbf{z}} h_{k}$ is easy to compute, and that is:

$$
\begin{aligned}
& \nabla_{\mathbf{z}} h_{k}=\left.\left[\begin{array}{lll}
\frac{\partial h}{\partial \mathbf{p}} & \frac{\partial h}{\partial \dot{\mathbf{p}}} & \frac{\partial h}{\partial \boldsymbol{\alpha}}
\end{array}\right]\right|_{\mathbf{z}=\mathbf{z}_{k \mid k-1}} \\
& \quad=\left[\begin{array}{lll}
-\mathbf{H}_{0} \mathbf{M}^{-1} \mathbf{K}, & -\mathbf{H}_{0} \mathbf{M}^{-1} \mathbf{C}, & -\mathbf{H}_{0} \mathbf{M}^{-1} \frac{\partial \mathbf{K}}{\partial \alpha} \mathbf{p}(k)-\mathbf{H}_{0} \mathbf{M}^{-1} \frac{\partial \mathbf{C}}{\partial \alpha} \dot{\mathbf{p}}(k)
\end{array}\right] .
\end{aligned}
$$

The idea of the EGDF algorithm is excited by the idea of the EKF algorithm, which is to linearize the non-linear state transmission Eq. (15) and measurement Eq. (16) with the first-order Taylor expansion to make the standard GDF algorithm suitable for identifying the augmented state and unknown input of the approximate linear dynamic system. It is obvious that the EGDF algorithm has a stricter mathematics derivation and observes the definitions of prior PDF (probability density function) and posterior PDF in Bayesian framework compared with the algorithm proposed by Lei et al. [19].

\section{Numerical studies}

In this section, two numerical examples are considered to evaluate the performance of the proposed EGDF method. The first example deals with a 3-DOF non-linear elastic structure, and the other one is to identify the non-linear parameters of a 4-DOF hysteretic structure.

\subsection{Example 1: 3-DOF non-linear elastic structure}

Consider a three-story non-linear elastic Duffing-type shear-beam building subject to unmeasured random excitation $u_{1}(t)$. The equations of motion are given by:

$$
\begin{aligned}
& {\left[\begin{array}{ccc}
m_{1} & 0 & 0 \\
m_{2} & m_{2} & 0 \\
m_{3} & m_{3} & m_{3}
\end{array}\right]\left\{\begin{array}{l}
\ddot{p}_{1}(t) \\
\ddot{p}_{2}(t) \\
\ddot{p}_{3}(t)
\end{array}\right\}+\left[\begin{array}{ccc}
c_{1} & -c_{2} & 0 \\
0 & c_{2} & -c_{3} \\
0 & 0 & c_{3}
\end{array}\right]\left\{\begin{array}{l}
\dot{p}_{1}(t) \\
\dot{p}_{2}(t) \\
\dot{p}_{3}(t)
\end{array}\right\}+\left[\begin{array}{ccc}
k_{11} & -k_{21} & 0 \\
0 & k_{21} & -k_{31} \\
0 & 0 & k_{31}
\end{array}\right]\left\{\begin{array}{l}
p_{1}(t) \\
p_{2}(t) \\
p_{3}(t)
\end{array}\right\}} \\
& +\left[\begin{array}{ccc}
k_{13} & -k_{23} & 0 \\
0 & k_{23} & -k_{33} \\
0 & 0 & k_{33}
\end{array}\right]\left\{\begin{array}{l}
p_{1}^{3}(t) \\
p_{2}^{3}(t) \\
p_{3}^{3}(t)
\end{array}\right\}=\left\{\begin{array}{c}
u_{1}(t) \\
0 \\
0
\end{array}\right\} .
\end{aligned}
$$

In which $p_{i}$ is the inter-story drift between $i$ th and $(i-1)$ th stories $(i=1,2,3)$, $m_{1}=m_{2}=m_{3}=1000 \mathrm{~kg}, c_{1}=c_{2}=c_{3}=0.6 \mathrm{kNs} / \mathrm{m}, k_{11}=120 \mathrm{kN} / \mathrm{m}, k_{21}=120 \mathrm{kN} / \mathrm{m}$, $k_{31}=60 \mathrm{kN} / \mathrm{m}, k_{13}=200 \mathrm{kN} / \mathrm{m}, k_{23}=200 \mathrm{kN} / \mathrm{m}$ and $k_{33}=-50 \mathrm{kN} / \mathrm{m}$. for the elastic structure with $k_{13}=k_{23}=k_{33}=0$, the natural frequencies are $f_{1}=0.73 \mathrm{~Hz}, f_{2}=1.74 \mathrm{~Hz}$ and $f_{3}=2.93 \mathrm{~Hz}$ with the corresponding damping ratios $\zeta_{1}=1.42 \%, \zeta_{2}=4.56 \%$ and $\zeta_{3}=5.08 \%$.

Assuming that four parameters including $c_{2}, c_{3}, k_{21}$ and $k_{31}$ are unknown to be determined, their initial values are $0.3 \mathrm{kN} \mathrm{s} / \mathrm{m}, 0.9 \mathrm{kN} \mathrm{s} / \mathrm{m}, 100 \mathrm{kN} / \mathrm{m}$ and $80 \mathrm{kN} / \mathrm{m}$, respectively. Thus, the augmented state vector is $\left\{p_{1}, p_{2}, p_{3}, \dot{p}_{1}, \dot{p}_{2}, \dot{p}_{3}, c_{2}, c_{3}, k_{21}, k_{31}\right\}^{T}$. Two signals are measured for 
identification, which are the accelerations of $\ddot{p}_{1}$ and $\ddot{p}_{2}$. Also $3 \%$ environment noise is added to the measured signals. Based on the proposed EGDF algorithm, the unknown random excitation, displacement, velocity and the unknown parameters are all identified. First, the identified excitation is plotted in Fig. 1.

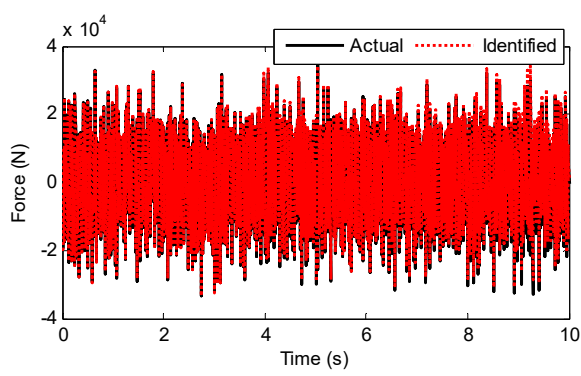

Fig. 1. The actual and identified results of random force $u_{1}$

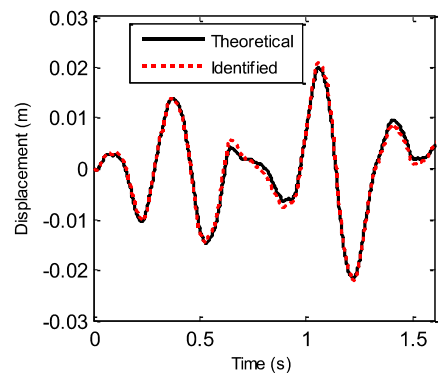

a)

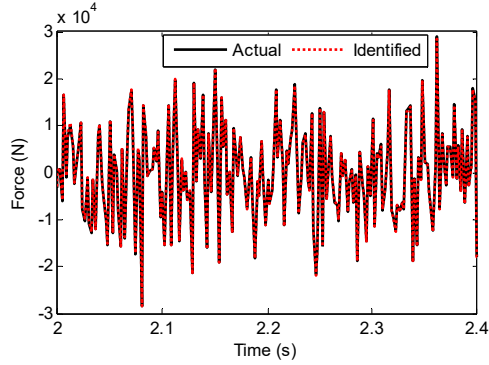

Fig. 2. Zoom-in view of a segment (2-2.4 s) of Fig. 1

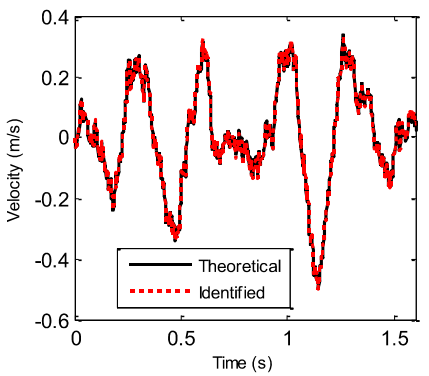

b)

Fig. 3. a) The theoretical and identified displacement at the 2nd floor,

b) the theoretical and identified velocity at the 2 nd floor

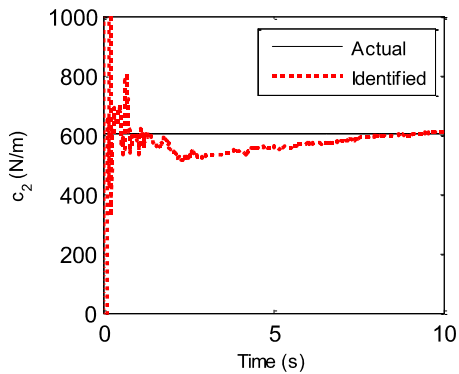

a)

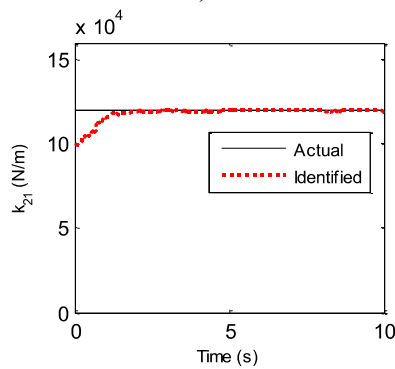

c)

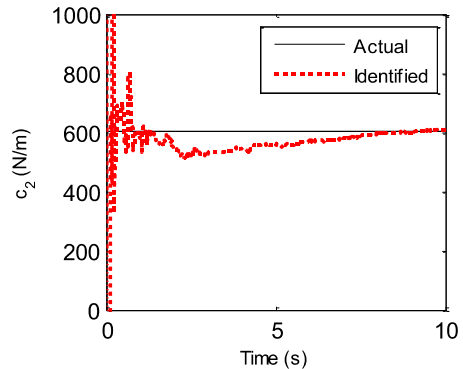

b)

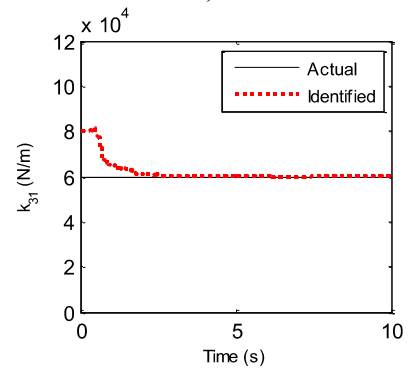

d)

Fig. 4. a) The actual and identified parameter value of $c_{2}$, b) the actual and identified parameter value of $c_{3}$, c) the actual and identified parameter value of $k_{21}$, d) The actual and identified parameter value of $k_{31}$ 
And to make a clearer comparison, the segment history of the actual and identified excitations from $2 \mathrm{~s}-2.4 \mathrm{~s}$ is also shown in Fig. 2. Then, the identified displacement and velocity responses at the 2nd floor are compared with their corresponding theoretical values as shown in Fig. 3. From Fig. 1 to Fig. 3, one can know that the identified force and structural state are both accurate. Also, the four identified parameters are plotted in Fig. 4. At the beginning of the identified procedure, the identified parameter values have the strong oscillatory, and then are quickly convergent to the neighborhoods of their corresponding actual values. From the above comparisons, it is clear that the proposed EGDF algorithm has the ability for simultaneous excitation and parameter identification for the non-linear Duffing-type system.

\subsection{Example 2: 4-DOF hysteretic structure with time-varying parameters}

The other numerical example is to identify the hysteretic parameters of a four-story hysteretic shear-beam building subject to unmeasured excitation on the top floor of the building, as shown by Fig. 5.

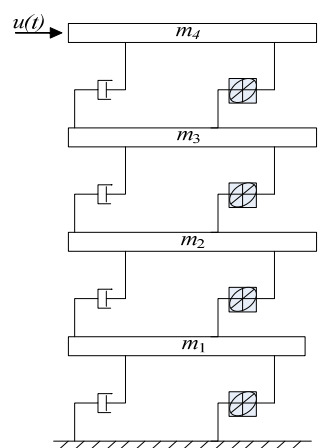

Fig. 5. A hysteric shear-frame building under unknown excitation

The equations of motion are given as:

$\mathbf{M} \ddot{\mathbf{p}}(t)+\mathbf{C} \dot{\mathbf{p}}(t)+\mathbf{K r}(t)=\mathbf{B}_{u} \mathbf{u}(t)$,

where $\mathbf{r}(t)$ is the vector of hysteretic force with $r_{i}(t)(i=1,2, \ldots, 4)$ being the $i$ th floor hysteretic restoring force and is herein modeled by the Bouc-Wen non-linear differential equation, which can be written as:

$\dot{r}_{i}=\theta_{i}\left(\dot{p}_{i}-\dot{p}_{i-1}\right)-\beta_{i}\left|\dot{p}_{i}-\dot{p}_{i-1}\right|\left|r_{i}\right|^{\alpha_{i}-1}-\gamma_{i}\left(\dot{p}_{i}-\dot{p}_{i-1}\right)\left|r_{i}\right|^{\alpha_{i}}$,

where $\theta_{i}, \beta_{i}, \gamma_{i}$ and $\alpha_{i}$ are the Bouc-Wen hysteretic parameters. The hysteretic force is hereditary, depending on the past history of deformation, and its description is very complicated. Thus, the structure is non-linear. Structural hysteretic performance can be used as the indicator of the development of structural damage under dynamic excitation. Therefore, structure damage can be detected based on the identification of hysteretic parameters.

The following parameters are used in the example: mass of each of each floor $m_{1}=m_{2}=m_{3}=m_{4}=500 \mathrm{~kg}$; each story stiffness $k_{1}=k_{2}=k_{3}=k_{4}=24 \mathrm{kN} / \mathrm{m}$; each story damping coefficients $c_{1}=c_{2}=c_{3}=c_{4}=0.1 \mathrm{kNs} / \mathrm{m}$; hysteretic parameters are time-varying, and have the relations as $\theta_{1}=\theta_{2}=\theta_{3}=\theta_{4}, \quad \beta_{1}=\beta_{2}=\beta_{3}=\beta_{4}, \quad \gamma_{1}=\gamma_{2}=\gamma_{3}=\gamma_{4}$, $\alpha_{1}=\alpha_{2}=\alpha_{3}=\alpha_{4}$. The external excitation on the 4 th floor is assumed to be a white noise force. The response time histories of the non-linear structure are calculated by the Runge-Kutta method. Three acceleration responses at the 1st, 3rd and 4th floors are assumed to be measured. The influence of measurement noise on identification is considered by superimposition of noise 
process with the computed response quantities. In this example, $3 \%$ RMS noise are adopted. The external excitation applied on the 4th floor is assumed to be unknown for identification.

In the EGDF algorithm, the unknown quantities to be identified are the augmented state vector $\mathbf{z}=\{\mathbf{p}, \dot{\mathbf{p}}, \mathbf{r}, \theta, \beta, \gamma, \alpha\}$ and the unmeasured external excitation $u(t)$. The sampling time interval is $0.5 \mathrm{~s}$. Based on the algorithm, the unmeasured excitation, displacement, velocity, hysteretic forces and hysteretic parameters can be identified. Fig. 6 shows the curves of the actual and identified excitations, and Fig. 7 is the segment view of Fig. 6 from $0 \mathrm{~s}$ to $40 \mathrm{~s}$, which is to make a clearer comparison. The identified structural state of the 2 nd floor is plotted in Fig. 8 compared with the responding theoretical value. The identified velocity response is very accurate, and the identified displacement response is also accurate with a minor error. The identified hysteretic force between 3rd and 4th floor is plotted in Fig. 9. The identified error occurs mainly due to the error of the identified displacement. The identified time-varying non-linear parameters are shown in Fig. 10. The dashed lines marked in red represent the up and bottom boundaries of the identified parameters. From all the above figures, it demonstrates that the proposed EGDF approach is capable of simultaneous excitation and parameter identification for non-linear Bouc-Wen hysteretic systems.

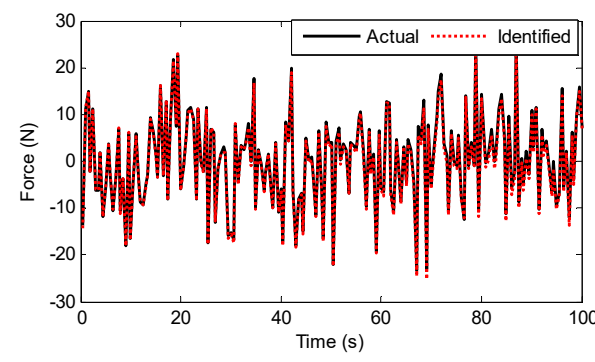

Fig. 6. The actual and identified results of random force $u(t)$

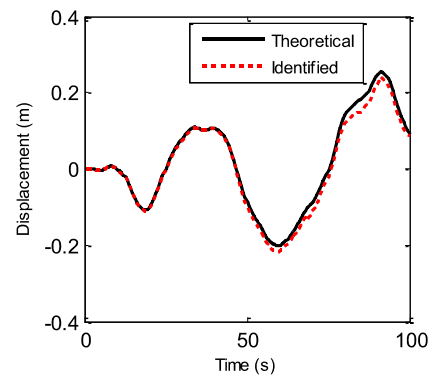

a)

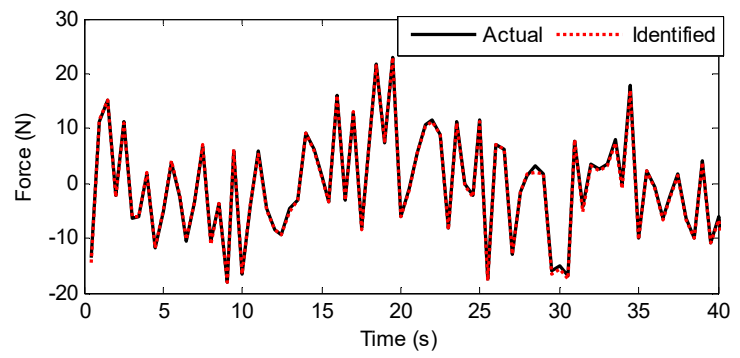

Fig. 7. Zoom-in view of a segment (0-40 s) of Fig. 6

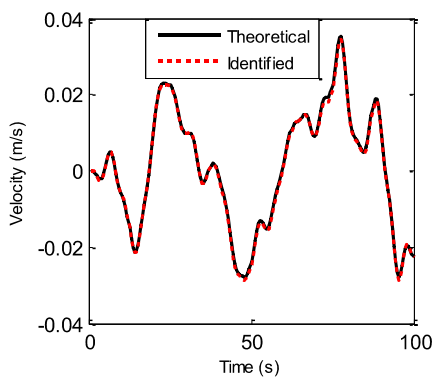

b)

Fig. 8. a) The theoretical and identified displacement of the vertical direction at the 2 nd floor, b) the theoretical and identified velocity of the vertical direction at the 2nd floor

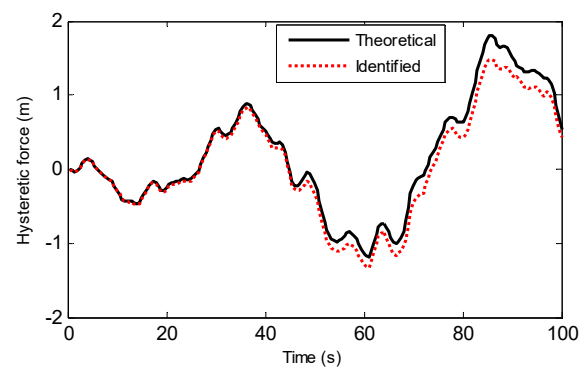

Fig. 9. The theoretical and identified hysteretic force between the 3rd and 4th floors 


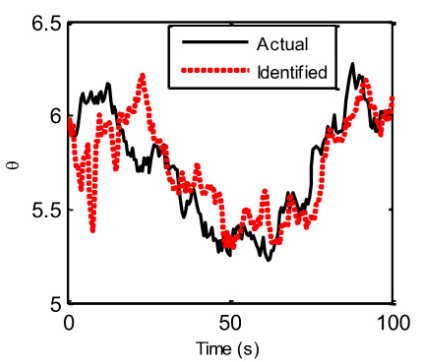

a)

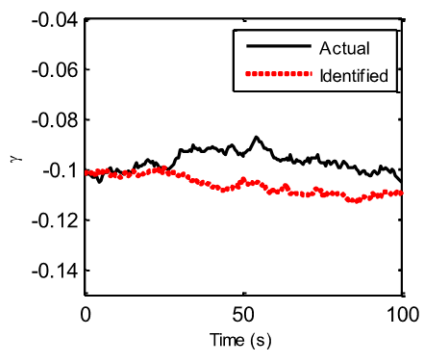

c)

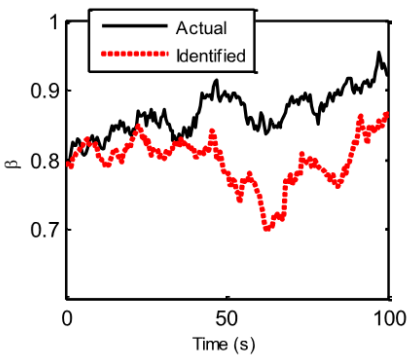

b)

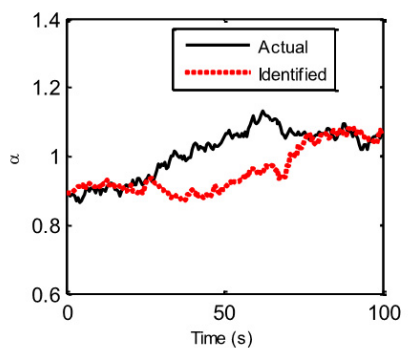

d)

Fig. 10. The actual and identified hysteretic parameters

Zhimin Wan extended the algorithm of the traditional GDF method. Ting Wang studied the algorithm of the the traditional GDF method. Qibai Huang provided the improved idea of the proposed method. Weiguang Zheng studied the extended EKF algorithm. Feng Gu helps to check the English.

\section{Conclusions}

In this paper, an algorithm is proposed for simultaneous excitation and parameter identification of non-linear structural systems. The approach is motivated by the linearization idea of EKF algorithm, and it is the extension version of the GDF algorithm for non-linear structural systems. The structural states and uncertain parameters are combined as the so-called augmented states. The original state transmission and measurement equations are both transformed into the non-linear equations due to the augmented states. The first-order Taylor expansion is adopted to linearize the non-linear system. With the linearization values of the actual and prior of augmented states, the EGDF method is derived similar to the GDF method. The only differences are the sensitivity matrices of the non-linear state transmission and measurement functions to the augmented states and forces. Numerical examples of two typical non-linear systems, including a three-story elastic Duffing-type shear-beam building and a four-story hysteretic shear-beam building, are conducted to identify unknown parameters and excitation, and the results demonstrate the effectiveness of the approach.

Additionally, it should be noted that the proposed EGDF algorithm is not applied for strong non-linear structures, such as Lorenz chaotic structures. This is because EKF approximates through the first-order linearization of both the non-linear state transmission and observation equations by Taylor series expansion. For strong non-linear structural systems, the unscented Kalman filter (UKF) or particle filter (PF) may be used for simultaneous excitation and parameter identification, which is our future work.

\section{Acknowledgements}

This work was supported by the National Natural Science Foundation of China (No. 51575201 
and No. 51405093) and the Technology Foundation of Nantong (GY12016043).

\section{References}

[1] Chang F. K. Structural health monitoring. Proceedings of the 6th and 7th International Workshops on Structural Health Monitoring, Stanford University, Stanford, CA, CRC Press, New York, 2009.

[2] Wu Z. S., Xu B., Harada T. Review on structural health monitoring for infrastructure. Journal of Applied Mechanics, Vol. 6, 2003, p. 1043-1054.

[3] Masri S. F., Smyth A. W., Chassiakos A. G., Caughey T. K., Hunter N. F. Application of neural networks for detection of changes in nonlinear systems. Journal of Engineering Mechanics, Vol. 126, 2000, p. 666-676.

[4] Masri S. F., Caffrey J. P., Caughey T. K., Smyth A. W., Chassiakos A. G. Identification of the state equation in complex non-linear systems. International Journal of Non-Linear Mechanics, Vol. 39, 2004, p. 1111-1127.

[5] Smyth A. W., Masri S. F., Kosmatopoulos E. B., Chassiakos A. G., Caughey T. K. Development of adaptive modeling techniques for non-linear hysteretic systems. International Journal of Non-Linear Mechanics, Vol. 37, 2002, p. 1435-1451.

[6] Hoshiya M., Saito E. Structural identification by extended Kalman filter. Journal of Engineering Mechanics, Vol. 110, 1984, p. 1757-1770.

[7] Ghanem R., Shinozuka M. Structural system identification. I: Theory. Journal of Engineering Mechanics, Vol. 121, 1995, p. 255-264.

[8] Shinozuka M., Ghanem R. Structural system identification. II: Experimental verification. Journal of Engineering Mechanics, Vol. 121, 1995, p. 265-273.

[9] Liu X., Escamilla-Ambrosio P. J., Lieven N. Extended Kalman filtering for the detection of damage in linear mechanical structures. Journal of Sound and Vibration, Vol. 325, 2009, p. 1023-1046.

[10] Yang J. N., Lin S., Huang H., Zhou L. An adaptive extended Kalman filter for structural damage identification. Structural Control and Health Monitoring, Vol. 13, 2006, p. 849-867.

[11] Petersen C. D., Fraanje R., Cazzolato B. S., Zander A. C., Hansen C. H. A Kalman filter approach to virtual sensing for active noise control. Mechanical Systems and Signal Processing, Vol. 22, 2008, p. 490-508.

[12] Sato T., Qi K. Adaptive Ho filter: its application to structural identification. Journal of Engineering Mechanics, Vol. 124, 1998, p. 1233-1240.

[13] Liu L., Lei Y., He M. A two-stage parametric identification of strong nonlinear structural systems with incomplete response measurements. International Journal of Structural Stability and Dynamics, 2015, p. 1640022.

[14] Yoshida I. Damage detection using Monte Carlo filter based on non-Gaussian noise. Structural Safety and Reliability, ICOSSAR'01, 2001.

[15] Khalil M., Sarkar A., Adhikari S., Poirel D. The estimation of time-invariant parameters of noisy nonlinear oscillatory systems. Journal of Sound and Vibration, Vol. 344, 2015, p. 81-100.

[16] Ching J., Beck J. L., Porter K. A. Bayesian state and parameter estimation of uncertain dynamical systems. Probabilistic Engineering Mechanics, Vol. 21, 2006, p. 81-96.

[17] Yang J. N., Pan S., Huang H. An adaptive extended Kalman filter for structural damage identifications II: unknown inputs. Structural Control and Health Monitoring, Vol. 14, 2007, p. 497-521.

[18] Lei Y., Jiang Y., Xu Z. Structural damage detection with limited input and output measurement signals. Mechanical Systems and Signal Processing, Vol. 28, 2012, p. 229-243.

[19] Lei Y., Wu Y., Li T. Identification of non-linear structural parameters under limited input and output measurements. International Journal of Non-Linear Mechanics, Vol. 47, 2012, p. 1141-1146.

[20] Gillijns S., De Moor B. Unbiased minimum-variance input and state estimation for linear discrete-time systems with direct feedthrough. Automatica, Vol. 43, 2007, p. 934-937.

[21] Anderson B. D., Moore J. B. Optimal Filtering. Prentice-Hall, Englewood Cliffs, NJ, 1979.

[22] Lourens E., Papadimitriou C., Gillijns S., Reynders E., Roeck De G., Lombaert G. Joint input-response estimation for structural systems based on reduced-order models and vibration data from a limited number of sensors. Mechanical Systems and Signal Processing, Vol. 29, 2012, p. 310-327. 


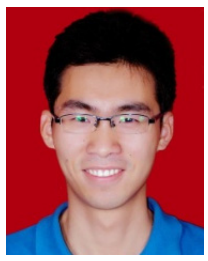

Zhimin Wan received the B.S. degree in mechanical engineering and automation from Hohai University, China, in 2010. He completed his Ph.D. in mechanical engineering from Huazhong University of Science and Technology, China, in 2015. Now he is a post doctor in Huazhong University of Science and Technology, China. His current research interests include structural dynamics, inverse problem, machine vision and intelligent drive.

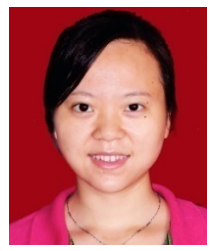

Ting Wang received the B.S. degree in mechanical engineering and automation from Hohai University, China, in 2010. She completed her Ph.D. in mechanical engineering from Huazhong University of Science and Technology, China, in 2015. Now she is a lecturer in School of Mechanical Engineering, Nantong Vocational University, China. Her current research interests include inverse problem and intelligent control.

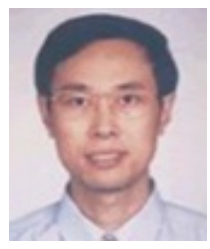

Qibai Huang received the M.S. degrees in vehicle engineering from Jilin University, China, in 1987, and completed his Ph.D. in mechanical vibration and Noise Control from Huazhong University of Science and Technology, in China, in 1994. Now he is a Professor in College of Mechanical Science and Engineering, Huazhong University of Science and Technology. His research interests include mechanical vibration, noise control, and vehicle dynamics.

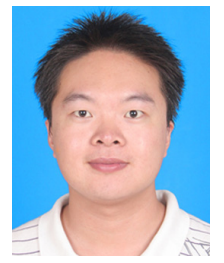

Weiguang Zheng received the B.S. degree in mechanical engineering and automation from Wuhan University, China, in 2005. He completed his Ph.D. in mechanical engineering from Huazhong University of Science and Technology, China, in 2013. Now he is a lecturer in School of Mechanical and Electrical Engineering, Guilin University of Electronic Technology, China. His current research interests include structural dynamics, smart structures and active/passive noise control.

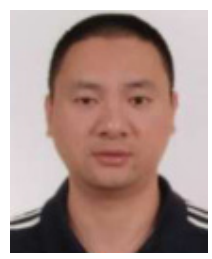

Feng Gu received the B.S. degree in mechanical engineering and automation from Southeast University, China, in 2000. Now he is an Associate Professor in School of Vehicle and Transportation Engineering, Nantong Vocational University, China. His current research interests include vehicle dynamics and composite material. 